\title{
PHASE CALIBRATORS FOR MERLIN
}

\author{
A.R. Patnaik, I.W.A. Browne, P.N. Wilkinson \\ University of Manchester, Nuffield Radio Astronomy Laboratories, Jodrell \\ Bank, Macclesfield, Cheshire SK11 9DL, UK. \\ J.M. Wrobel \\ National Radio Astronomy Observatory, P.O. Box O, Socorro, New \\ Mexico 87801, USA.
}

\begin{abstract}
The VLA was used to derive a catalogue of phasereference sources for MERLIN. These sources can also be used for other interferometer arrays such as the VLBA. The positions of the sources in the catalogue are repeatable to about 5 milliarcsec rms.
\end{abstract}

\section{INTRODUCTION}

The MERLIN array has so far been almost exclusively used as a hybrid mapping instrument, enabling only relatively strong, point-dominated sources to be mapped using the closure technique. MERLIN is currently being upgraded in several important areas, including the addition of a new $32 \mathrm{~m}$ antenna at Cambridge, a new broad-band correlator system with full polarisation capabilities, and cooled receivers (Wilkinson, this volume). The sensitivity of the upgraded MERLIN is expected to be about $3 \mathrm{mJy}$ per baseline in a 1-minute integration at $5 \mathrm{GHz}$. The capabilities of MERLIN can be further enhanced by phase-reference observations, which will permit a substantial increase in the coherent integration time on target sources. This will enable MERLIN to map sources with peak brightnesses of a few mJy per beam area at 1.6 and $5 \mathrm{GHz}$. Thus, sources mappable with the VLA could also be mapped with MERLIN, with its higher angular resolution of 50 milliarcsec (mas) at $5 \mathrm{GHz}$ and 200 mas at $1.6 \mathrm{GHz}$. In addition, phase-referencing fixes the positions of the MERLIN maps, something which will be inceasingly important in the era of the Hubble Space Telescope. MERLIN has a resolution intermediate between the VLA and the EVN and thus will provide unique information about a wide range of radio sources.

\section{CHARACTERISTICS OF PHASE CALIBRATORS}

We have carried out test phase-reference observations with MERLIN at 1.6 $\mathrm{GHz}$ and our results indicate that: 
1. It is desirable to have a phase calibrator within about $2^{\circ}$ of the target source because MERLIN's phase stability at $1.6 \mathrm{GHz}$ is dominated by the ionosphere. The temporal variation of phase indicates that the calibrator should be observed frequently, with a cycle time $\leq 5$ minutes.

2. The phase calibrator should have $\geq 50 \mathrm{mJy}$ correlated flux density on all baselines and should have simple structure (point or point-like) which can be mapped to recover the 'structure phase'.

3. The positional accuracy of the phase calibrator should be as high as possible for astrometric reasons and to maximise the coherent integration time.

\section{WHERE TO FIND THEM?}

In general, the flat spectrum radio sources satisfy the above requirements, and can be considered as promising phase calibrator candidates. To find flat spectrum sources with the required density on the sky, sources must be selected down to about $200 \mathrm{mJy}$ at $5 \mathrm{GHz}$. We have derived a list of phase calibrator candidates from the Green Bank surveys at 1.4 and $5 \mathrm{GHz}$ (Condon et al. 1985,1986 and 1989) by using the software package DAOPHOT available in STARLINK. We selected 905 sources with the following criteria.

1. $\mathrm{S}(5 \mathrm{GHz}) \geq 200 \mathrm{mJy}$,

2. Spectral index flatter than 0.5 ,

3. $35^{\circ} \leq \delta \leq 75^{\circ}$ and,

4. $|b| \geq 2.5^{\circ}$.

Since the positional accuracies of the Green Bank surveys (20-30 arcsec) were not adequate for our purposes, and also the structures of most of the sources were not known, we decided to measure source positions and structures using the VLA.

\section{VLA OBSERVATIONS}

Observations were carried out in the A configuration at $8.4 \mathrm{GHz}$ with a bandwidth of $25 \mathrm{MHz}$ using 23 antennas. We used phase calibrators from the VLBI calibrator list of $\mathrm{Ma}$ et al. (1990), for which the positional accuracies are good to one mas. Each target source was observed for 2 minutes including antenna drive times, giving an on-source integration time of about 1 to 1.5 minutes. Phase calibrators were observed after every two target sources. The total observing time was 60.5 hours. We observed the group of sources around $3 \mathrm{C} 345$, whose positions are known from the VLBI list, to determine the positional accuracies of our observations. Also, to check the repeatability of our measurements we reobserved some sources. In general, the data were of good quality except for a few hours at the beginning of our run which were affected by a snow storm. The sources observed during the storm were reobserved later in the programme. 
After careful calibration of the data, images were made using the a simple procedure involving two cycles of phase self-calibrations. The resulting images had typical rms noise levels of $0.2 \mathrm{mJy}$ per beam area, or a dynamic range of about 1000:1. The positions were measured before self-calibration, from either the first dirty map or the first clean map.

\section{RESULTS}

Of the 905 sources, $84 \%$ have a total flux density at $8.4 \mathrm{GHz} \geq 100 \mathrm{mJy}$ and also have $\geq 80 \%$ of that flux density in a compact component. These sources are suitable phase calibrators for MERLIN. We find the following positional accuracies:

a) from the repeated sources, an rms of 5 mas,

b) from the 3C 345 group of sources, an rms somewhat better than 5 mas,

c) from the data affected by the snow storm, an rms of 18 mas.

It is our intention to make this list of MERLIN phase calibrators available to the radio astronomical community through dBase IV. This work is in progress and will be reported in more detail elsewhere.

\section{ACKNOWLEDGEMENTS}

We are grateful to Dr. J. Condon for supplying us with the Green Bank survey tapes and to Dr. T. Muxlow for help in phase-calibration tests with MERLIN.

\section{REFERENCES}

Condon, J.J. and Broderick, J.J. 1985, Astr. J., 90, 2540. Condon, J.J. and Broderick, J.J. 1986, Astr. J., $\underline{91}, 1051$.

Condon, J.J., Broderick, J.J. and Seielstad, G.A. 1989, Astr. J., $\underline{97}, 1064$. $\mathrm{Ma}$, C., Shaffer, D.B., de Vegt, C., Johnston, K.J. and Russel, J.L. 1990, Astr. J., $\underline{99}, 1284$. 